\title{
Experimental Assessment
}

\section{e Trigeminal Neuralgia Induced by Cobra Venom in the Rat Leads to Deficits in Abilities of Spatial Learning and Memory}

Zhe Wu, MD ${ }^{1}$, Xiao-yan Qian, CRNA ${ }^{1}$, Jian-xiong An, MD, PhD ${ }^{1}$, Cai-cai Liu, MD ${ }^{1,2}$, Ming Tian, $\mathrm{PHD}^{3}$, Doris K. Cope, $\mathrm{MD}^{4}$, and John P. Williams, MD ${ }^{4}$.

From: ${ }^{1}$ Department of Anesthesiology, Pain Medicine

\& Critical Care Medicine

Aviation General Hospital of China Medical University, Beijing, China; ${ }^{2}$ Department of Anesthesiology, Weifang Medical University, Weifang

City, Shandong Province, China; ${ }^{3}$ Beijing Friendship Hospital, Capital Medicine University, Beijing, China; ${ }^{4}$ Department of Anesthesiology, University of Pittsburgh School of Medicine, Pittsburgh, PA

Address Correspondence: Jian-xiong An, MD, PhD Prof. \& Chair

Dept. of Anesthesiology, Pain Medicine \& CCM

Aviation General Hospital of

China Medical Universty \& Beijing Institute of Translational

Medicine

Chinese Academy of Sciences Beiyuan Rd.3\#, Beijing, 100012, China E-mail:

anjianxiong@yahoo.com

Disclaimer: Xiao-yan Qian and Zhe Wu contributed equally to this work.

Manuscript received: 09-26-2017 Revised manuscript received: $11-25-2014$

Accepted for publication: 12-02-2014

Free full manuscript: www.painphysicianjournal.com
Background: Patients with chronic pain usually suffer from cognitive impairment, with memory deterioration being the most common deficit that affects daily functioning and quality of life. The causes for this impairment are not clear despite intensive clinical studies. Few studies have evaluated impaired learning using animal models of persistent pain.

Objective: In this study, a new trigeminal neuralgia model induced by cobra venom was adopted to explore effects of chronic pain on spatial learning and memory in rats.

Study Design: Controlled animal study.

Setting: Department of Anesthesiology, Pain Medicine \& Critical Care Medicine, Aviation General Hospital of China Medical University.

Methods: Thirty adult male Sprague-Dawley rats were randomly divided into 2 groups $(n=15)$ : NS control group and cobra venom group, $0.9 \%$ sterile saline or cobra venom solution was injected into the sheath of the infraorbital nerve (ION), respectively. The development of trigeminal neuralgia was accessed by changes in free behavioral activity 3 days before the surgery and 3, 7, 12, 20, and 30 days after the surgery to identify whether the model was successful or not. Morris water maze test determined the abilities of spatial learning and memory at the time points before the surgery, and 2 weeks and 5 weeks after the surgery. We also observed the ultrastructure of the ION and medulla oblongata of rats following 8 weeks of chronic trigeminal neuropathic pain.

Results: Rats with the cobra venom injection displayed significantly more face grooming and fewer exploratory activities compared to the NS control group or baseline $(P<0.01)$. Both groups improved their latency to reach the platform with the largest difference on the first day $(P<0.01)$, but without memory deficits in a probe trial for the second water maze protocol. For the third water maze testing, the rats in the cobra venom group experienced decreased abilities of spatial learning and memory, a longer latency with spatial memory deficits during the probe trial $(P<0.05)$. At the ultrastructural level, we found changes in the medulla oblongata after cobra venom injection resulting in severe demyelination and loss of axons that might be implicated in the causes of cognitive deficits.

Limitations: Limitations include partial vision loss in the eye on the lesion side of the rats that might be missed and the absence of evaluating the ultrastructural changes in other parts of the brain.

Conclusions: The results of this study suggest that trigeminal neuralgia induced by cobra venom in adult rats can impair spatial learning and memory function over time and results in demonstrable changes in the ultrastructure of the medulla oblongata. This new animal model may be useful for future studies on the effect of chronic pain on learning and cognition.

Key words: Cognitive deficits, memory deterioration, cobra venom, trigeminal neuralgia, electron microscopy

Pain Physician 2015; 18:E207-E216 
ognitive impairments are a commonly observed clinical manifestation in chronic pain patients. These deficits are present in a wide range of cognitive functions such as memory $(1,2)$, attention (3), and decision-making (4). Memory deterioration is the most common cognitive deficit reported in chronic pain patients $(2,5-7)$. Although many clinical investigations have studied the etiology of memory deficits in chronic pain patients, results have been both contradictory and inconclusive. Some studies indicate that memory impairment may be associated with the intensity of pain (5), however, in experimental settings, pain minimally affects cognitive ability in healthy individuals (8). Additionally, several studies in patients with chronic pain indicate that the prolonged use of opioids can cause cognitive changes $(9,10)$, while numerous others report the opposite effect in pain patients with improvement in cognitive functioning (11).

The etiology of cognitive deficits is unclear, but changes have been shown in the following: morphometry and neurotransmitter changes in related brain areas $(12,13)$, reduced sleep quality (14), long-term use of medication (15), pain interference in sensory processing (3), as well as psychological symptoms (e.g., stress, anxiety, and depression) $(5,16)$. Given the remarkable and well-established role that stress plays in the onset of some mood-related alterations, some authors suggest that cognitive deficits may be due to the effects of pain-induced chronic stress $(5,17,18)$. Another possible explanation is that the ongoing demand for attention that pain causes, limits mental resources for processing and task performance (19). Evidence supporting an underlying neurobiological basis for cognitive dysfunction in chronic pain patients is based on neuroimaging studies in chronic pain patients. These studies show reduced gray matter and abnormal connectivity in brain regions, such as the anterior cingulate and prefrontal cortices $(13,20)$. Similar anatomical results were obtained in a rat model of neuropathic pain. Rats had reduced gray matter in frontal cortical regions compared to sham controls, 6 months after nerve injury (21).

An adequate understanding of the effects of pain on memory requires the use of animal models in which variables can be more easily controlled to understand underlying neural mechanisms. Previous commonly used animal pain models include the spared nerve injury (SNI) model and the complete Freund's adjuvant (CFA) model. The former consists of ligation and transection of the tibial and common peroneal branches of the sciatic nerve while sparing the sural nerve (22). The latter is an inflammatory pain model induced by injecting CFA in the tibio-tarsal joint (23). However, persistent pain in the lower limbs may damage an animal's locomotor ability, thus influencing experimental outcomes. An et al (24) developed a new neuropathic pain model induced by administration of cobra venom to the infraorbital nerve (ION) in the rat producing characteristics similar to that of trigeminal neuralgia. The experimental procedure is easy to perform, and rats exhibit stable mechanical allodynia within the distribution of the ION. Here, we applied this new animal model to explore the effects of chronic pain on cognitive function.

In this study, we explored spatial learning and memory performance using the Morris water maze task after cobra venom-induced trigeminal neuralgia in rats, comparing changes in memory before and after the operation in each group and between groups. In addition, we observed the ultrastructure of the ION and medulla oblongata of rats following 8 weeks of chronic pain and compared these to control animals.

\section{Methods}

\section{Subjects}

All the experiments were done in accordance to IASP guidelines on the use of awake animals with efforts made to minimize the number of animals and their discomfort (25). Adult, male, Sprague-Dawley rats weighing between 180 - $200 \mathrm{~g}$ provided by the Laboratory Animal Center of the Academy of Military Medical Sciences were used in this experiment. Rats were housed under controlled conditions (temperature of $22 \pm 22^{\circ} \mathrm{C}$ and 12:12 light-dark cycle, lights on at 8 a.m.) with food and water available ad libitum. Thirty animals were randomly assigned to either the cobra venom group or sham-operated group. The cobra venom solution or $0.9 \%$ sterile saline injection, respectively, was injected under direct vision into the sheath of the ION.

\section{Drug Preparation}

The lyophilized cobra venom (Formosan cobra; Sigma, St. Louis, MO) was dissolved in $0.9 \%$ sterile saline. A volume of $4 \mu \mathrm{L}$ saline containing $0.4 \mathrm{mg}$ lyophilized cobra venom was administrated to each model rat.

\section{Experimental Procedure}

The cobra venom model was established as described previously (24). Rats were lightly anesthetized with sodium pentobarbital $(40 \mathrm{mg} / \mathrm{kg}$ intraperitoneally). An incision was made about one $\mathrm{cm}$ in length 
radically above the superciliary arch to expose the left infraorbital nerve, then cobra venom was injected into the sheath of the ION. The sham-operated group underwent exposure of the left infraorbital nerve with injection of saline. The incision was closed using 5-0 absorbable sutures. Rats with obvious loss of vision in the eye on the lesion side were excluded. There were 13 rats left in each group.

\section{Behavioral testing}

Rats were tested 3 days before the surgery and $3,7,12,20$, and 30 days after the surgery. Testing was conducted in a darkened room (light provided by a 60 $W$ red light bulb suspended one meter above the observation area) with a $45 \mathrm{~dB}$ background noise. Rats were placed in a transparent plastic cage $(24 \times 35 \times 18 \mathrm{~cm})$ with a mirrored back. A video camera was placed one meter in front of the cage. The rat's behavior was videotaped for a duration of 7 minutes, starting 15 minutes after placement in the cage. Videotaped behavior was analyzed offline by an experimenter who was blind to the condition of the rat. Behavioral changes of the rats observed by video recordings, the number and duration of face grooming (movement patterns in which paws contact facial areas), and exploratory (walking, running, climbing, rearing) behaviors were analyzed to confirm the presence of pain behavior.

\section{Morris Water Maze: Learning and Memory Testing}

The Morris water maze (MWM) generally consists of a black circular pool (180 cm diameter, $60 \mathrm{~cm}$ high) filled with water $\left(30 \mathrm{~cm}\right.$ depth) at $19^{\circ} \mathrm{C}-22^{\circ} \mathrm{C}$ and virtually divided into 4 equivalent quadrants. An escape platform ( $10 \mathrm{~cm}$ diameter) submerged $2 \mathrm{~cm}$ beneath the water surface was placed in the middle of one of the quadrants. The platform was painted black as was the pool wall and floor to make it invisible in the water. The pool was surrounded by several distal visual cues in the walls.

Rats received 4 acquisition trials per day for 4 consecutive days. Each day, a trial was initiated by placing each rat into the water facing the pool wall from one of the 4 pre-determined positions. If animals failed to find the platform within 90 seconds, they were picked up and placed directly on it for 30 seconds before a new trial (inter-trial interval 30 seconds). The platform location remained in the same position throughout all trials and days, but the starting direction differed with each trial, each day. Swim speeds and latency to reach plat- form were video recorded. On the fifth day ( 24 hours following the last hidden platform trial), a probe trial was conducted in which the platform was removed from the pool to measure spatial bias for the previous platform location. We placed each rat in the pool in the quadrant opposite to the trained platform location and tracked the rat for 60 seconds, measuring the percentage of time spent in the previous target quadrant as well as the number of crossings over the previous platform location.

The WMW task was performed 3 times at the time points before the surgery, and 2 weeks and 5 weeks after the surgery. All procedures were exactly the same except for the location of the platform. During the second WMW testing, the platform was placed in the opposite quadrant from the first testing. For the third test period, the platform was placed back in the initial quadrant.

\section{Electron Microscopy}

Histological analysis of 2 rats from each group was performed 8 weeks after surgery. Animals were deeply anesthetized and perfused via the left ventricle with normal saline, followed by fixation with $4 \%$ paraformaldehyde and $2 \%$ glutaraldehyde $(\mathrm{pH} 7.4)$. The left ION and medulla oblongata tissues was dissected out after one hour at $4^{\circ} \mathrm{C}$, bathed for 2 hours in $3 \%$ glutaraldehyde, then washed in $0.1 \mathrm{M}$ phosphate buffer (PB) for 3 times and finally post-fixed in 1\% osmium tetroxide for 2 hours. After washing, specimens were dehydrated in graded ethanol, infiltrated and flatembedded using Epon, and polymerized for 2 - 3 days at $60^{\circ} \mathrm{C}$. The sections were stained with uranyl acetate and lead citrate and ultrathin sections (50 - $70 \mathrm{~nm}$ ) were cut and collected on copper grids for an electron microscope.

\section{Statistical analysis}

Results were expressed as mean \pm standard deviation (SD). Differences between groups were calculated using repeated measures 2-way ANOVA with post hoc test (Bonferroni's test) to analyze behavioral alterations and latencies to reach the platform in the water maze. Mean comparisons for data about percentage of time spent in the platform quadrant and platform crossings were carried out with one-way ANOVA for the 2 groups. Swim speeds, percentage of time spent in the platform quadrant, and platform crossings between groups were analyzed using student's t test. Data analysis was performed using the statistical software 
SPSS 13.0 and significant differences were determined as $P<0.05$.

\section{Results}

\section{Behavioral Changes of Trigeminal Neuralgia Model}

Animals were first videotaped to determine if pain behavior was present in the experimental model. As shown in Fig. 1, rats with a cobra venom injection displayed significantly more face grooming frequency (F1, $24=109.9, P<0.01)$ and time $(F 1,24=374.12, P<$ 0.01 ) compared to control rats (Fig. $1 \mathrm{~A}, \mathrm{~B}$ ), which is the most obvious behavioral change occurring following the onset of trigeminal neuralgia in rats. In addition, frequency $(F 1,24=42.83, P<0.01)$ and duration of exploratory $(\mathrm{F} 1,24=175.81, P<0.01)$ behavior showed a consistent decreasing trend over time. (Fig. 1C, D).

\section{Morris Water Maze}

\section{Spatial Learning}

The protocol tested in the water maze task contained both learning and memory (probe) trials. Learning phases are presented first. No differences were found between latencies to reach the platform and swim speed before the pain model was established, demonstrating that there were no pre-existing differences between the groups $(P>0.05)$ (Fig. 2A). During the second test period, the swim speed between con-

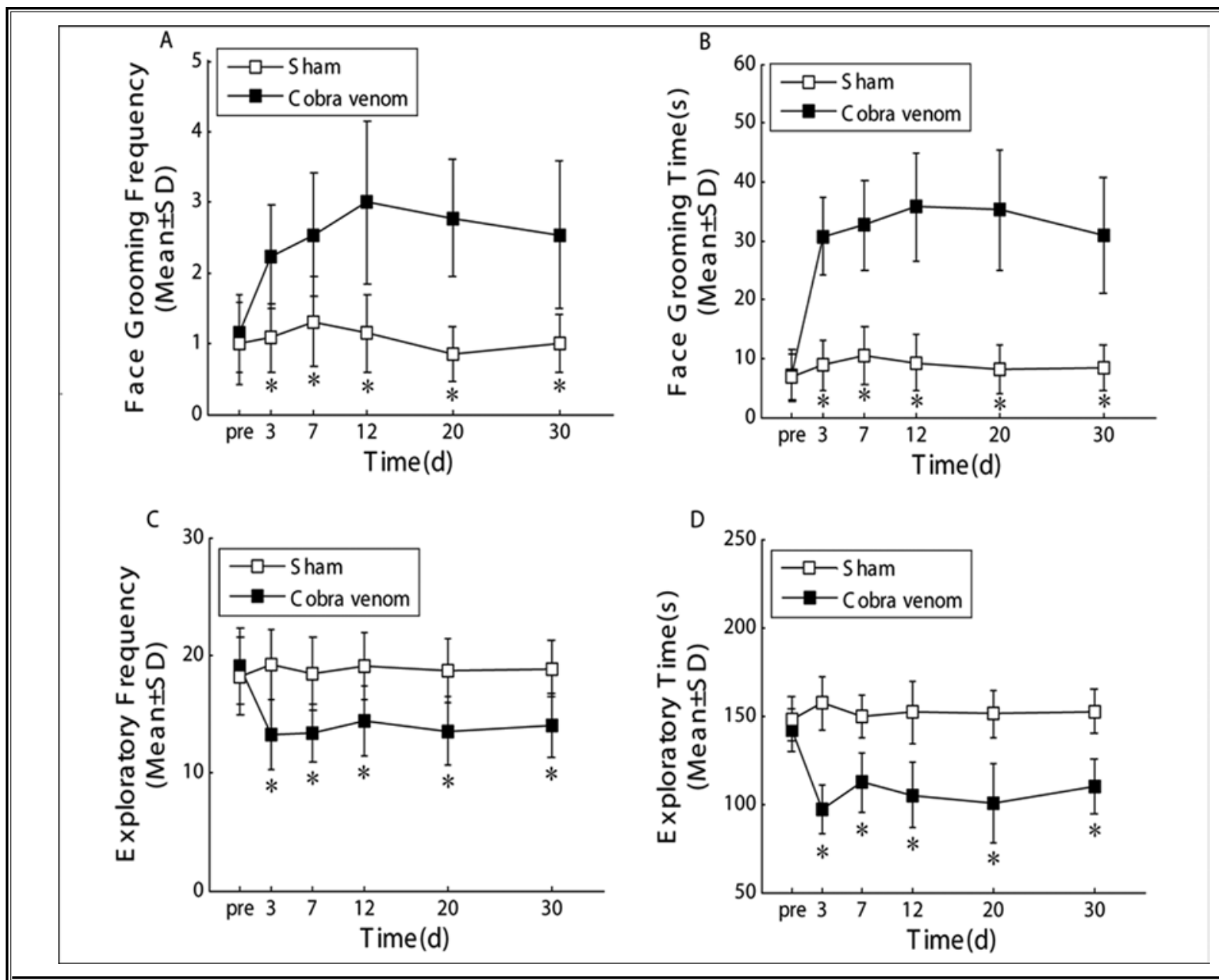

Fig. 1. Frequency and duration of observed face-grooming and exploratory behavior during a 7 min observation session before the operation (pre) and at 5 postoperative time points. (A-D) No significant baseline differences between groups were found before the operation $(P>0.05)$. Behavioral activity showed significant differences between groups after the operation. Significance is defined as ${ }^{*} P<0.05$ compared to the sham group at each point in time $(P<0.05)$. 
Trigeminal Neuralgia Induced by Cobra Venom in Rats
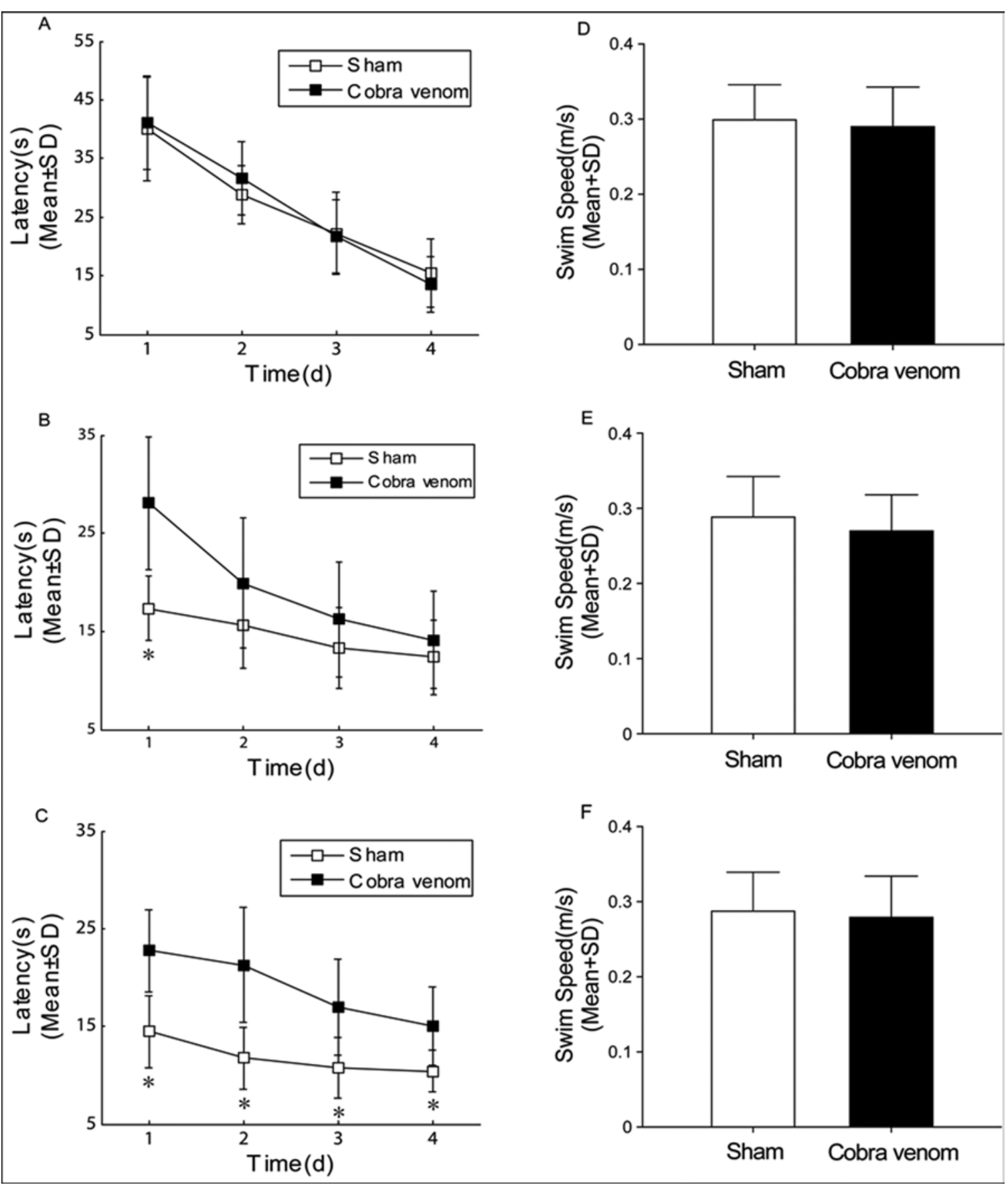

Fig. 2. Comparison of water maze performance in animals. (A) No differences were found in the latency curve between the two groups before the model was established. (B) 2 weeks after the surgery, the latency curve showed major difference between groups on day 1 , both groups displayed similar latencies to reach the platform after training. (C) 5 weeks after the surgery, the latency curve showed a slower learning rate in the cobra venom group. (D.E.F) No significant differences were found between the swimming speeds before or after the model was established. Significance is defined as $* P<0.05$ compared to the sham group at each point in time $(P<0.05)$. 
trol and cobra venom rats did not differ (Fig. 2E). Both groups improved their latency to reach the platform, and a significant difference between groups was found (F1, $24=14.82, P<0.05)$ with the largest difference on the first day $(P<0.01)$ (Fig. 2B). In a third experiment we analyzed if the long-term existence of neuropathic pain would affect a rat's performance in the water maze task. We used the same protocol as described above with the same set of animals. Swim speed was similar between groups (Fig. 2F), but the latency curve showed a slower learning rate in the cobra venom rats (F1, $24=76.54, P<0.01$ ) (Fig. 2C).

\section{Spatial Memory: Probe Trials}

On probe trials, percentages of the total probe trial time in the target quadrant and platform crossings showed differences only on probe trial 3 between groups ( $P<0.01$ ) (Fig. 3). Comparisons among probe trials of the cobra venom group showed decreased av- erage percentages of the total probe trial time in the target quadrant $(F 2,36=4.201, P=0.023)$, on probe trial 3 the decrease was significant $(P<0.05)$ (Fig. 3A). Platform site crossings showed a similar pattern with reduced site crossings in the third trials (F2, $36=4.284$, $P=0.021$ ) (Fig. 3B). No difference was found among probe trials of the sham group.

\section{Ultrastructure Changes of Rat's ION and Medulla Oblongata}

At 8 weeks after surgery, uranyl acetate and lead citrate staining were performed to investigate the ION and medulla oblongata of each group. For the cobra venom group, ultrastructural analysis in cross-section of the rat's nerve after cobra venom injection showed prominent abnormal features with severe demyelination and diminution of axons (Fig. 4). The structure of myelin was disturbed and the concentric layers expanded. For the sham group, the myelinated axon

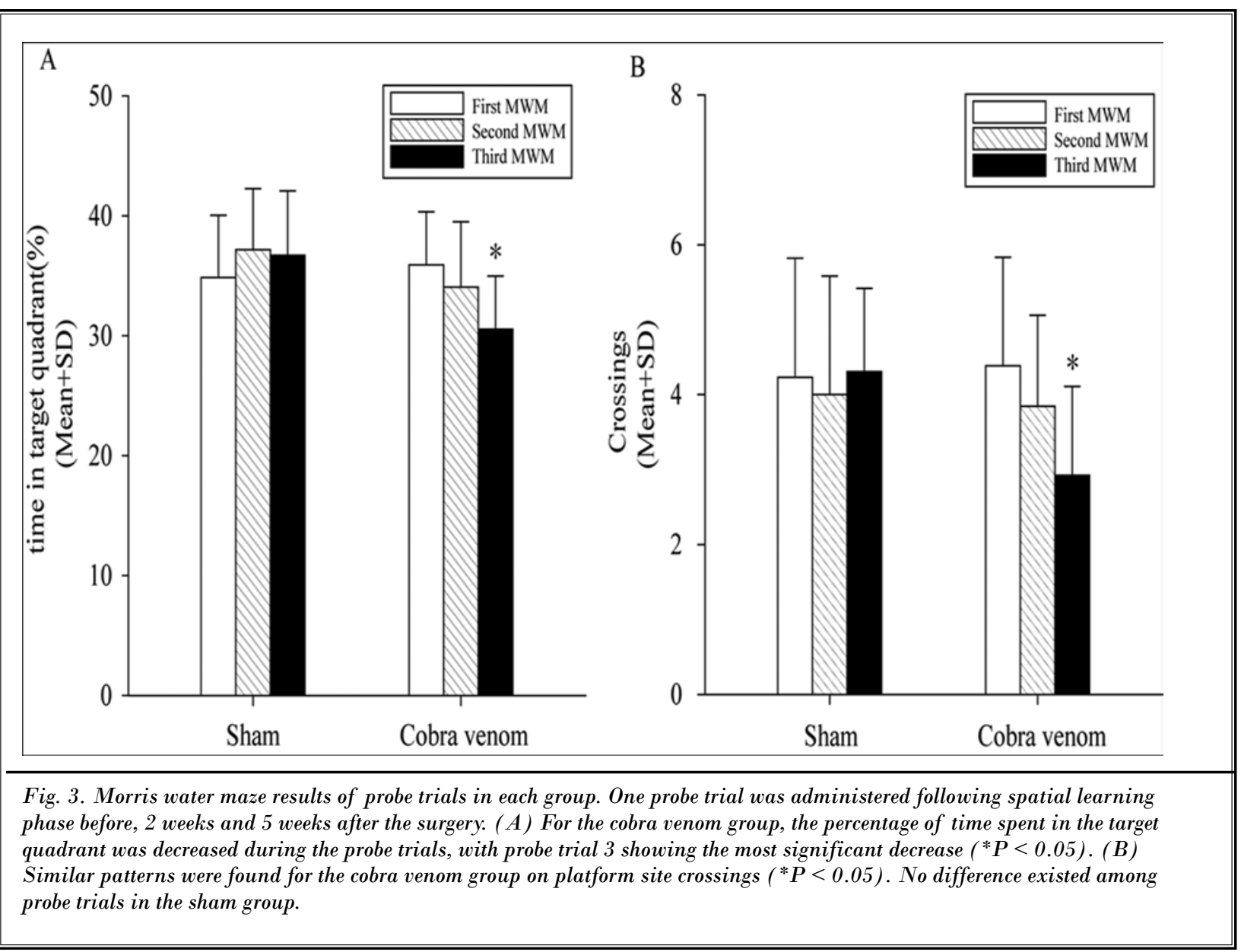




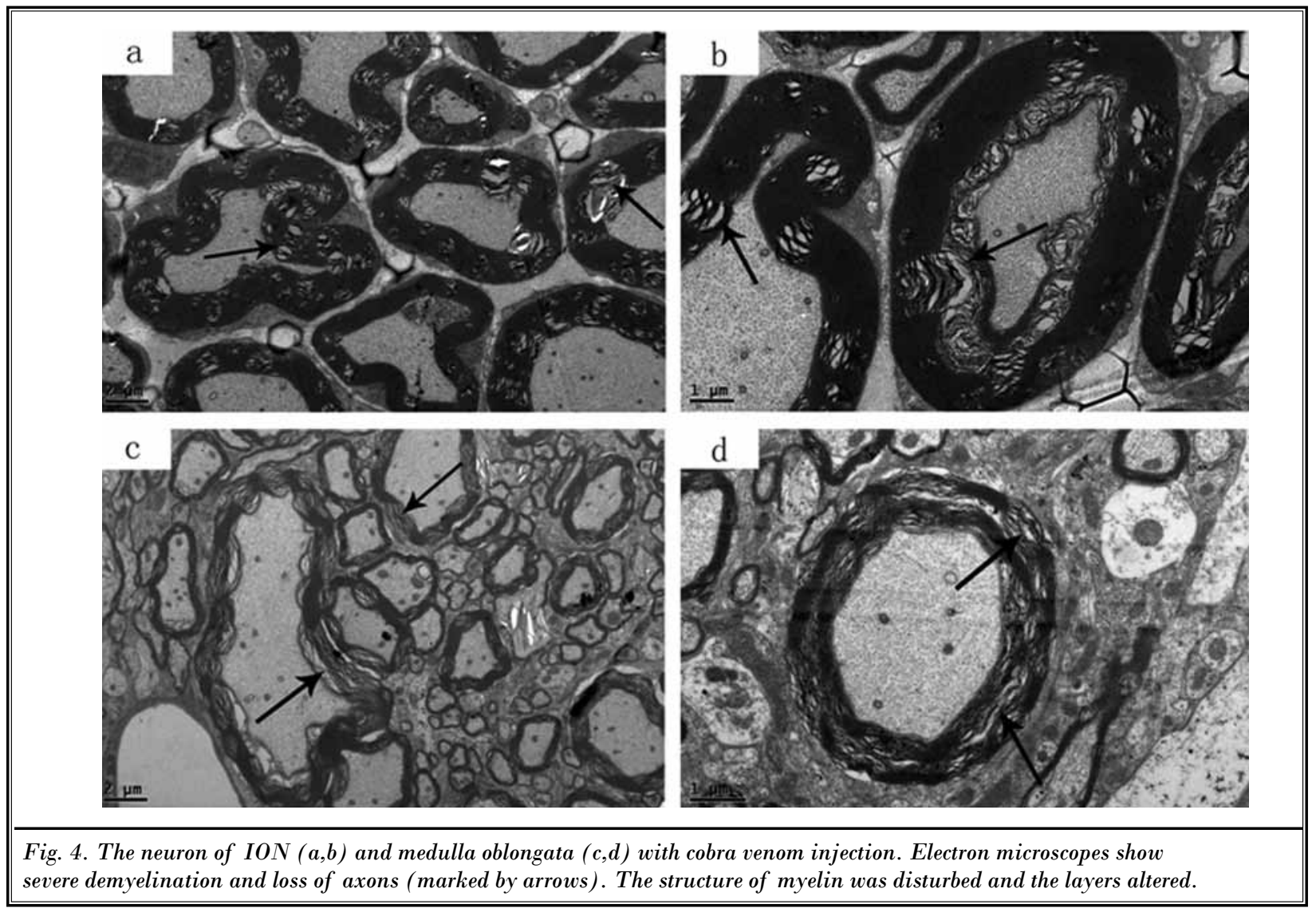

appeared as a nearly circular profile surrounded by a spirally wound multilamellar sheath with compact and uniform structure. Myelin sheaths were essentially the same thickness and the structure of layers was clear and dense (Fig. 5).

\section{Discussion}

In human patients with chronic pain (usually defined as pain lasting more than 6 months) memory deficits that affect daily functioning and quality of life are frequently encountered. Compared to humans, each rat month has been calculated to be roughly equivalent to 2.5 human years (26). In the present study we examined the effects of persistent neuropathic pain on cognitive functioning in a new rat model induced by cobra venom at time periods beyond the acute pain phase and comparable to years of pain experience in humans.

In behavioral change tests, rats manifest significant changes in free behavioral activity. In the $1-30$ day postoperative period, rats groom the territory of the injured nerve frequently and for long periods and they show a profound decrease in exploratory behav- ior. In previous studies $(27,28)$, the most remarkable behavioral change following ION ligation is a dramatic increase in face-grooming activity; other changes include increase in freezing-like behavior and decrease in exploratory behavior. Our results are consistent with these findings, indicating that the model induced by cobra venom causes a condition similar to trigeminal neuropathic pain.

In our study, we have demonstrated that chronic trigeminal neuralgia disrupts the function of spatial learning and memory with a significant time effect, which is similar to observations in human chronic pain patients with deficits in tasks involving attention and memory (29). For the second water maze protocol, both groups improved their latency to reach the platform with the largest difference on the first day, indicating that chronic pain led to learning dysfunction of the rats. However, repeated training reversed this impairment in memory acquisition. No spatial memory deficits were found during the probe trial. Escape latency indicates the ability of spatial information acquired, while probe time relates to the retention and retrieval of acquired 

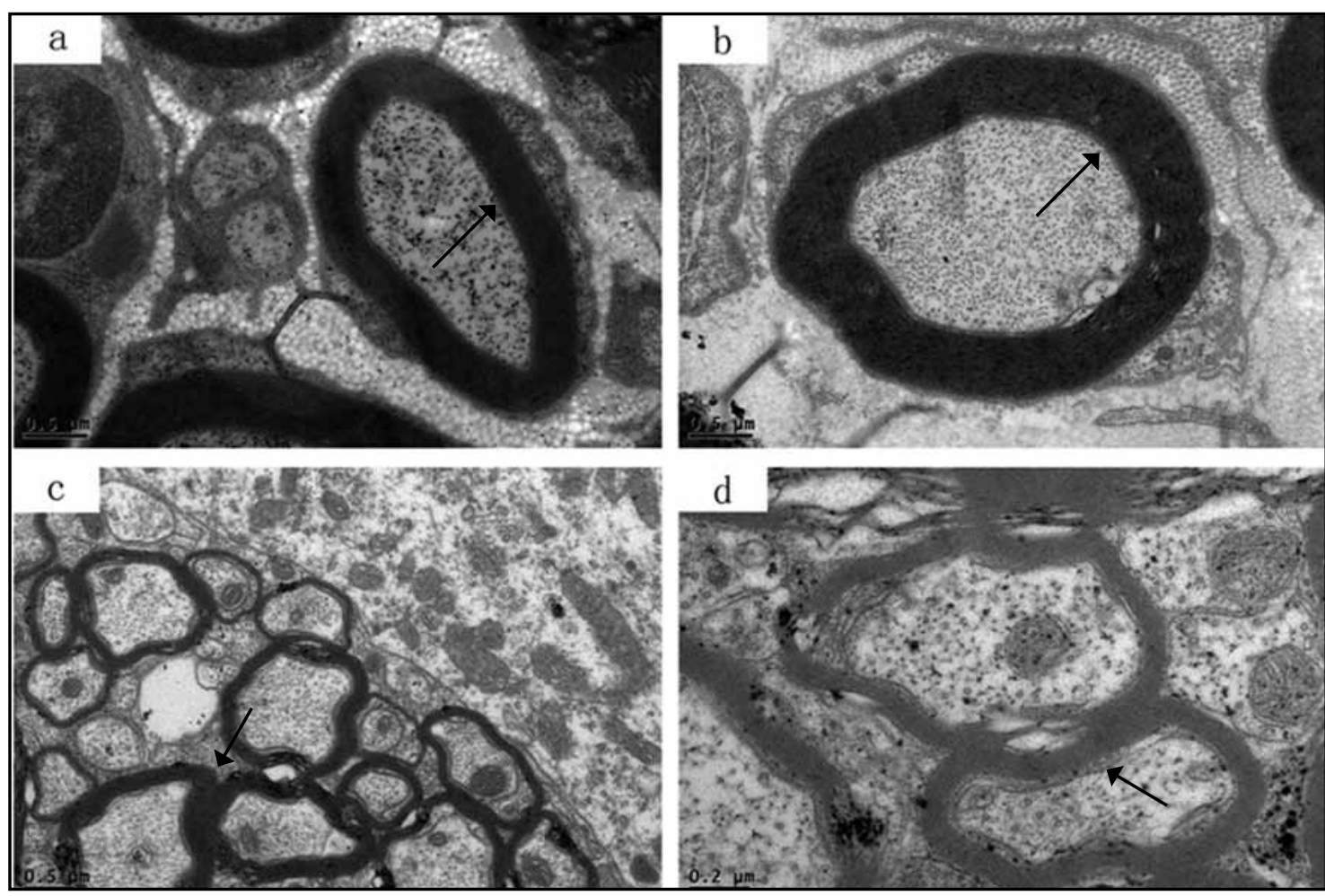

Fig. 5. The normal neuron of ION $(a, b)$ and medulla oblongata $(c, d)$. Electron microscopes show the structure of myelin sheaths and axon is entire (marked by arrows).

memories. Some studies show that repeated neuropsychological function testing could improve the results to a certain extent. Radak et al (30) suggest swimming training improves cognitive function in rats, with parallel attenuation of the accumulation of oxidatively damaged proteins. Exercise in the Morris water maze can upregulate the expression of brain-derived neurotrophic factor (BDNF) mRNA and protein, thus improve the ability of learning and memory (31). For the third set of tests, the latency curve showed a slower learning rate in the cobra venom group associated with spatial reference memory deficits during the probe trial. These findings suggest that long-term maintenance of neuropathic pain may affect the function of spatial learning and memory in the water maze task. These results are consistent with reports that spatial memory is reduced in spared nerve injury (32) or spinal nerve ligation models of neuropathic pain (33). Throughout the experiment, swim speeds were similar between groups, showing that pain-induced motor impairment was not the cause for the differences found in the acquisition period of the water maze.
We think that brainstem is primarily the pathway that transmits peripheral pain signals to brain related areas, so we first studied the medulla oblongata to observe the ultrastructural changes during long-time chronic trigeminal neuralgia. In the study, the ultrastructural changes of the ION and medulla oblongata after cobra venom injection reveal severe demyelination and diminution of axons. Hilton et al (34) first observed focal loss of myelin and close apposition of demyelinated axons with trigeminal neuralgia caused by vascular compression. However, the mechanisms associated with the ultrastructural changes in the medulla oblongata after administration of cobra venom are poorly understood. The effects of the main ingredients in cobra venom used in the model are cardiotoxin (CTX), which induces conduction blockade of the nerves, and phospholipase A2 (PLA2), which hydrolyzes phospholipids in the axonal membrane with the aid of CTX. CTX highly binds with the protein layer surrounding axons, thus resulting in changes of the membrane structure (35). In a previous study, we observed an inflammatory response within the distribution of the rat's ION (24). 
It is uncertain whether the same reaction exists by cobra venom transmission along the nerve to the central nervous system, which may contribute to the observed changes of the medulla oblongata. Another possible mechanism of this phenomenon may involve signal conduction of noxious stimuli to the central nervous system after trigeminal nerve lesion. Benoist et al (36) recorded neuronal electrophysiological activities in the dorsal thalamus and the somatosensory cortices using the $\mathrm{CCl}$ model. They found that stimuli of the contralateral to the injured nerve also resulted in abnormal electrophysiological changes in cerebral nuclei mentioned above, but with a less degree than ipsilateral, and the possible explanation might be that nerve injury caused by chronic constriction could also lead to lesion of the high nerve center.

Some authors include chronic pain as a type of degenerative neurological disease. This conclusion is based on data that chronic pain is processed by a complex neuronal network that involves both alterations to cognitive capacities in addition to neuroanatomical changes (37). A large amount of research demonstrates functional and structural central nervous system changes in patients with chronic pain $(20,37,38)$. A meta-analysis that evaluated 152 studies of acute and chronic pain found that several brain regions were consistently activated during pain perception with the most prominent difference being that chronic pain more often activates brain regions involved in cognitive and/or emotional pain processing (39). Studies have shown that the brainstem is involved in chronic pain processing in humans $(40,41)$. RodriguezRaecke et al (42) studied 32 patients with chronic pain caused by primary hip osteoarthritis by using 3 Tesla MRI and found decreased gray matter in the anterior cingulate cortex (ACC), right insular cortex, dorsolateral prefrontal cortex, amygdala, and brainstem compared with control patients. Schmidt-Wilcke et al (43) found similar changes in the brainstem and somatosensory cortex. Given that peripheral neuropathic pain is shown to contribute to structural alterations in central neurons that are known to be involved in pain processing accompanying cognitive impairment in our study, we suggest that the changes could be implicated in the onset of cognitive deficits.

In our study, we determined whether the procedure resulted in vision loss in the eye on the lesion side of the rats only by naked-eye observation, partial loss of vision might have been missed. Otherwise, it should be noted that this study has examined the medulla oblongata only by electron microscope, it is not clear whether these changes of demyelination and loss of axons are only localized in the medulla oblongata, or if they are more of a generalized phenomenon. Therefore, further studies evaluating other parts of the brain, especially the frontal lobe which is directly responsible for short-term and long-term memory, may contribute to further understanding of the etiology of cognitive impairment in patients with chronic pain.

\section{Conclusion}

We conclude that chronic trigeminal neuralgia in rats impairs spatial learning and memory function over time and results in demonstrable changes in the ultrastructure of the medulla oblongata. The model of trigeminal neuralgia induced by cobra venom in the rat exhibits not only cutaneous hypersensitivity but also cognitive deficits similar to those evinced by human pain patients. The changes caused by this model suggest that it may be particularly useful for future studies on the effect of chronic pain on learning and cognition.

\section{References}

1. Brown SC, Glass JM, Park DC. The relationship of pain and depression to cognitive function in rheumatoid arthritis patients. Pain 2002; 96:279-284.

2. Grace GM, Nielson WR, Hopkins M, Berg MA. Concentration and memory deficits in patients with fibromyalgia syndrome. J Clin Exp Neuropsychol 1999; 21:477-487.

3. Eccleston C. Chronic pain and distraction: An experimental investigation into the role of sustained and shifting attention in the processing of chronic persistent pain. Behav Res Ther 1995;
33:391-405.

4. Apkarian AV, Sosa $Y$, Krauss BR, Thomas PS, Fredrickson BE, Levy RE, Harden RN, Chialvo DR. Chronic pain patients are impaired on an emotional decisionmaking task. Pain 2004; 108:129-136.

5. Hart RP, Martelli MF, Zasler ND. Chronic pain and europsychological functioning. Neuropsychol Rev 2000; 10:131-149.

6. Kewman DG, Vaishampayan N, Zald D, Han B. Cognitive impairment in musculoskeletal pain patients. Int J Psychiatry Med 1991; 21:253-262.
7. Landro NI, Stiles TC, Sletvold H. Memory functioning in patients with primary fibromyalgia and major depression and healthy controls. J Psychosom Res 1997; 42:297-306.

8. Veldhuijzen DS, Kenemans JL, de Bruin CM, Olivier B, Volkerts ER. Pain and attention: Attentional disruption or distraction?] Pain 2006; 7:11-20.

9. Sjogren $P$, Olsen AK, Thomsen AB, Dalberg J. Neuropsychological performance in cancer patients: The role of oral opioids, pain and performance status. Pain 2000; 86:237-245. 
10. Schiltenwolf $M$, Akbar M, Hug A, Pfuller U, Gantz S, Neubauer E, Flor H, Wang HL. Evidence of specific cognitive deficits in patients with chronic low back pain under long-term substitution treatment of opioids. Pain Physician 2014; 17:9-19.

11. Jamison RN, Schein JR, Vallow S, Ascher S, Vorsanger GJ, Katz NP. Neuropsychological effects of long-term opioid use in chronic pain patients. J Pain Symptom Manage 2003; 26:913-921.

12. Grachev ID, Fredrickson BE, Apkarian AV. Brain chemistry reflects dual states of pain and anxiety in chronic low back pain. J Neural Transm 2002; 109:1309-1334.

13. Schmidt-Wilcke T, Leinisch E, Straube A, Kampfe N, Draganski B, Diener HC, Bogdahn U, May A. Gray matter decrease in patients with chronic tension type headache. Neurology 2005; 65:1483-1486.

14. McCracken LM, Iverson GL. Predicting complaints of impaired cognitive functioning in patients with chronic pain. J Pain Symptom Manage 2001; 21:392-396.

15. O'Neill WM, Hanks GW, White L, Simpson $P$, Wesnes K. The cognitive and psychomotor effects of morphine in healthy subjects: A randomized controlled trial of repeated (four) oral doses of dextropropoxyphene, morphine, lorazepam and placebo. Pain 2000; 85:209-215.

16. Breslau N, Andreski P. Migraine, personality, and psychiatric comorbidity. Headache 1995; 35:382-386.

17. Hart RP, Wade JB, Martelli MF. Cognitive impairment in patients with chronic pain: The significance of stress. Curr Pain Headache Rep 2003; 7:116-126.

18. Radanov BP, Dvorak J, Valach L. Cognitive deficits in patients after soft tissue injury of the cervical spine. Spine 1992; 17:127-131.

19. Eccleston C, Crombez G. Pain demands attention: A cognitive-affective model of the interruptive function of pain. Psychol Bull 1999; 125:356-366.

20. Apkarian AV, Sosa Y, Sonty S, Levy RM, Harden RN, Parrish TB, Gitelman DR. Chronic back pain is associated with decreased prefrontal and thalamic gray matter density. J Neurosci 2004; 24:10410-10415.

21. Seminowicz DA, Laferriere AL, Millecamps M, Yu JC, Coderre TJ, Bushnell MC. MRI structural brain changes as- sociated with sensory and emotional function in a rat model of long-term neuropathic pain. Neurolmage 2009; 47:1007-1014.

22. Decosterd I, Woolf CJ. Spared nerve injury: An animal model of persistent peripheral neuropathic pain. Pain 2000; 87:149-158.

23. Butler SH, Godefroy F, Besson JM, WeilFugazza J. A limited arthritic model for chronic pain studies in the rat. Pain 1992; 48:73-81.

24. An JX, He Y, Qian XY, Wu JP, Xie YK, Guo QL, Williams JP, Cope Dk. A new animal model of trigeminal neuralgia produced by administration of cobra venom to the infraorbital nerve in the rat. Anesth Analg 2011; 113:652-656.

25. Zimmermann M. Ethical guidelines for investigations of experimental pain in conscious animals. Pain 1983; 16:109-110.

26. Andrello NA, Santos EF, Araújo MR, Lopes LR. Rat's age versus human's age: What is the relationship? Arq Bras Cir Dig 2012; 25:49-51.

27. Vos BP, Hans G, Adriaensen H. Behavioral assessment of facial pain in rats: Face grooming patterns after painful and non-painful sensory disturbances in the territory of the rat's infraorbital nerve. Pain 1998; 76:173-178.

28. Yeomans DC, Klukinov M. A rodent model of trigeminal neuralgia. Methods Mol Biol 2012; 851:121-131.

29. Dick BD, Rashiq S. Disruption of attention and working memory traces in individuals with chronic pain. Anesth Analg 2007; 104:1223-1229.

30. Radák Z, Kaneko T, Tahara S, Nakamoto H, Pucsok J, Sasvári M, Nyakas C, Goto $\mathrm{S}$. Regular exercise improves cognitive function and decreases oxidative damage in rat brain. Neurochemistry Int 2001; 38:17-23.

31. Adlard PA, Perreau VM, Engesser-Cesar C, Cotman CW. The timecourse of induction of brain-derived neurotrophic factor mRNA and protein in the rat hippocampus following voluntary exercise. Neuroscience Letters 2004; 363:43-48.

32. Leite-Almeida $\mathrm{H}$, Almeida-Torres $\mathrm{L}$, Mesquita AR, Pertovaara A, Sousa N, Cerqueira JJ, Almeida A. The impact of age on emotional and cognitive behaviors triggered by experimental neuropathy in rats. Pain 2009; 144:57-65.

33. Hu Y, Yang J, Hu Y, Wang Y, Li W. Ami- triptyline rather than lornoxicam ameliorates neuropathic pain-induced deficits in abilities of spatial learning and memory. Eur J Anaesthesiol 2010; 27:162-168.

34. Hilton DA, Love S, Gradidge T, Coakham HB. Pathological findings associated with trigeminal neuralgia caused by vascular compression. Neurosurgery 1994; 35:299-303.

35. Chang CC, Chuang ST, Lee CY, Wei JW. Role of cardiotoxin and phospholipase $A$ in the blockade of nerve conduction and depolarization of skeletal muscle induced by cobra venom. $\mathrm{Br}$ J Pharmacol 1972; 44:752-764.

36. Benoist JM, Gautron M, Guilbaud G. Experimental model of trigeminal pain in the rat by constriction of one infraorbital nerve: Changes in neuronal activities in the somatosensory cortices corresponding to the infraorbital nerve. Exp Brain Res 1999; 126:383-398.

37. Gracely RH, Geisser ME, Giesecke T, Grant MA, Petzke F, Williams DA, Clauw DJ. Pain catastrophizing and neural responses to pain among persons with fibromyalgia. Brain 2004; 127:835-843.

38. Maihofner $C$, Handwerker HO, Neundorfer B, Birklein F. Cortical reorganization during recovery from complex regional pain syndrome. Neurology 2004; 63:693-701.

39. May A. Neuroimaging: Visualising the brain in pain. Neurol Sci 2007; 28:S101-S107.

40. Zambreanu L, Wise RG, Brooks JC, lannetti GD, Tracey I. A role for the brainstem in central sensitisation in humans. Evidence from functional magnetic resonance imaging. Pain 2005; 114:397-407.

41. Hirakawa N, Tershner SA, Fields HL, Manning $\mathrm{BH}$. Bi-directional changes in affective state elicited by manipulation of medullary pain-modulatory circuitry. Neuroscience 2000; 100:861-871.

42. Rodriguez-Raecke R, Niemeier A, Ihle $\mathrm{K}$, Ruether W, May A. Brain gray matter decrease in chronic pain is the consequence and not the cause of pain. J Neurosci 2009; 29:13746-13750.

43. Schmidt-Wilcke T, Leinisch E, Ganssbauer S, Draganski B, Bogdahn U, Altmeppen J, May A. Affective components and intensity of pain correlate with structural differences in gray matter in chronic back pain patients. Pain 2006; 125:89-97. 